\title{
Measurement result of slice positioning sound of MRI equipment
}

\author{
Kenji Muto ${ }^{1, *}$, Kazuo Yagi ${ }^{2}$, Kentaro Eguchi ${ }^{2}$, Guoyue $\mathrm{Chen}^{3}$ and Kunihiko Takano ${ }^{1}$ \\ ${ }^{1}$ Tokyo Metropolitan College of Aeronautical Engineering, \\ Minamisenju 8-52-1, Arakawa-ku, Tokyo, 116-8523 Japan \\ ${ }^{2}$ Tokyo Metropolitan University, \\ Higashiogu 7-2-10, Arakawa-ku, Tokyo, 116-8551 Japan \\ ${ }^{3}$ Akita Prefectual University, \\ Ebinokuchi 84-4, Tsuchiya, Honjo, 015-0055 Japan
}

(Received 7 October 2005, Accepted for publication 10 November 2005)

Keywords: Magnetic resonance imaging, Noise level, Operating sound, Nonmagnetic element PACS number: 43.50.+y [DOI: 10.1250/ast.27.174]

\section{Introduction}

Magnetic resonance imaging (MRI) examinations are necessary and indispensable in medical diagnosis. The generated operation sound is loud during image acquirement. This loud sound generated during operation often makes patients uncomfortable. The A-weighted sound pressure level of the sound has been reported to be $100 \mathrm{dBA}$ or more [1-3]. Whileit is known that the examination room is subjected to a strong magnetic field environment, the noise level has not been fully investigated. We measured the operating sound of five imaging methods of MRI [3]. We found that the sound produced during the T2 weighted imaging method (T2W) is the loudedamong the five imaging methods. We also found, however, that the sound of slice positioning has also not been investigated yet. Slice positioning is used to decide the region of the human body targeted by the MRI diagnosis. Slice positioning is an imaging method used to decide the range of imaging in a short time. Therefore, it requires the use of a high-speed imaging method. It is executed for 20-60 seconds at the beginning of an MRI examination.

In this study, we measured the slice positioning sound with nonmagnetic microphones. We here report the measurement results of the noise distribution on the patient's table during slice positioning. We hope this data will be useful for the improvement of the MRI environment.

\section{Measurement conditions of MRI operation sound}

The following equipment was used for the measurement and analysis [3]: sixteen nonmagnetic electret condenser microphones (ECM), 11-030 series, made by AZDEN, eight microphone amplifiers AT-MA2 made by Audio Technica, a calibrator NC-74 (calibration level $94 \mathrm{~dB}$, calibration signal frequency $1,000 \mathrm{~Hz}$ ) made by RION, a sixteen-channel digital tape recorder SIR-1000 made by SONY (sampling frequency $48 \mathrm{kHz}$ ), and the FFT analyzer CF-5220 made by Ono Sokki for FFT and one-third octave analysis. The MRI equipment was Signa LX $1.5 \mathrm{~T}$ for the whole body made by the GE Yokogawa Medical Systems.

The signal from each microphone was recorded by the digital tape recorder. Each microphone was adjusted with a

\footnotetext{
*e-mail: muto@kouku-k.ac.jp
}

calibrator. Sixteen microphones were fixed to the wooden array with a plastic clip, as shown in Fig. 1. The ECM was composed of a polyester diaphragm, brass back plate, and a case of ABS and aluminum. These ECMs are omnidirectional microphones. These microphones, set at the height of $16 \mathrm{~cm}$, were arrayed on the patient's table $3 \mathrm{~cm}$ to the left of the center of the table, as shown in Fig. 2.

Each signal was corrected by the frequency characteristic of corresponding microphone, and calculated relative to the calibration signal level $(94 \mathrm{~dB}, 1,000 \mathrm{~Hz})$. One-third octave analysis was conducted using an FFT analyzer, where, the data length was 4,096, the window function in time domain was a Hanning window, and the frequency weighting characteristic for auditory sensation was the A weighting characteristic.

The imaging method for slice positioning was the echo planner method with a single echo. The duration of slice positioning was 50 seconds. We analyzed the sound that occurred during 100 seconds, in which the slice positioning was repeated twice. The equivalent continuous A-weighted sound pressure level during the 100 seconds was calculated. Additionally, the sound produced by typical imaging methods, T1W and T2W, was analyzed. The equivalent continuous A-weighted sound pressure levels during 60 seconds were calculated. Table 1 shows the conditions of these imaging methods. TR (repetition time), TE (echo time), frequency matrix, and phase matrix are conditions that govern the performance of the image.

\section{Sound of slice positioning of MRI equipment}

We show the noise level distribution on the patient's table using a measurement system with nonmagnetic microphones. The sound level of MRI was measured during an operating for obtaining an image of a phantom. The phantom is a cylinder of a mock human body made of sulfated nickel solution. The A-weighted sound pressure levels during slice positioning, $\mathrm{T} 1 \mathrm{~W}$ and $\mathrm{T} 2 \mathrm{~W}$, within the range of $3 \mathrm{~m}$ on the patient's table are as shown in Fig. 3. The sound pressure levels at the center and at the edge of the gantry were high. The value (103.4 dBA) of the slice positioning level at $0 \mathrm{~m}$ was greater than the value (101.4 dBA) of the T2W level at $0 \mathrm{~m}$. The value (103.1 dBA) of the slice positioning level at $1 \mathrm{~m}$ was smaller than the value $(104.8 \mathrm{dBA})$ of the T2W level at $1 \mathrm{~m}$. The 


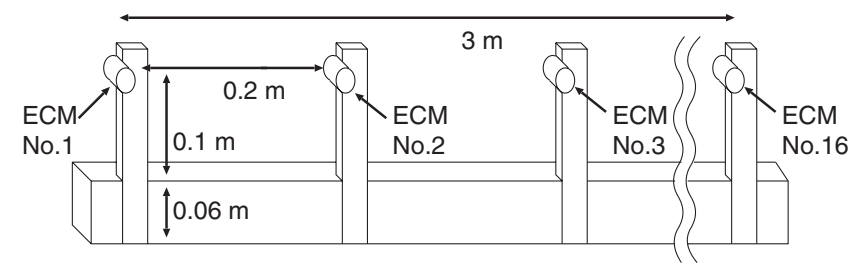

Fig. 1 Microphone array using sixteen ECMs.

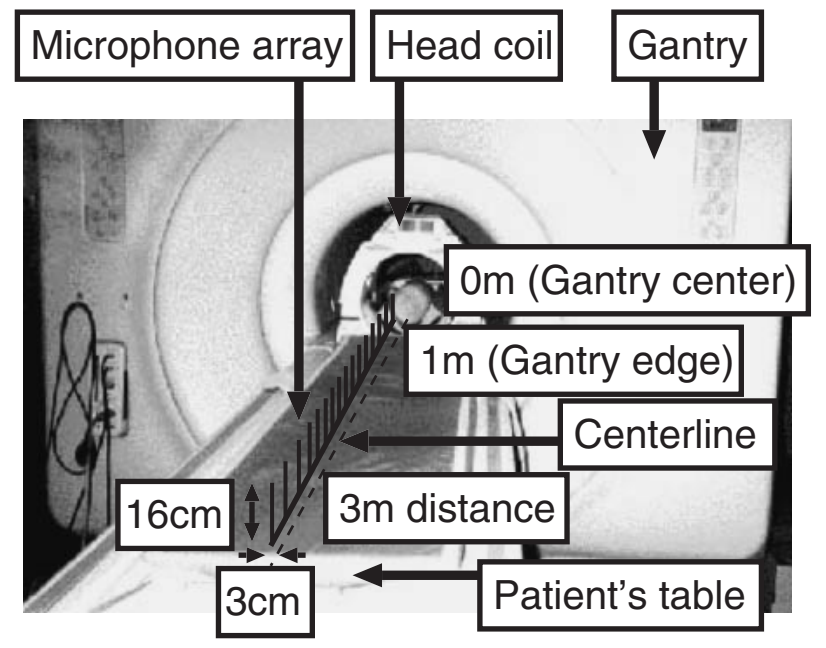

Fig. 2 MRI equipment and microphone array on the patient's table.

Table 1 Imaging conditions with phantom.

\begin{tabular}{crrc}
\hline & T1W & T2W & Slice positioning \\
\hline TR [ms] & 450 & 4,000 & 46 \\
TE [ms] & 20 & 102 & 1.6 \\
Frequency matrix & 256 & 256 & 256 \\
Phase matrix & 192 & 192 & 192 \\
\hline
\end{tabular}

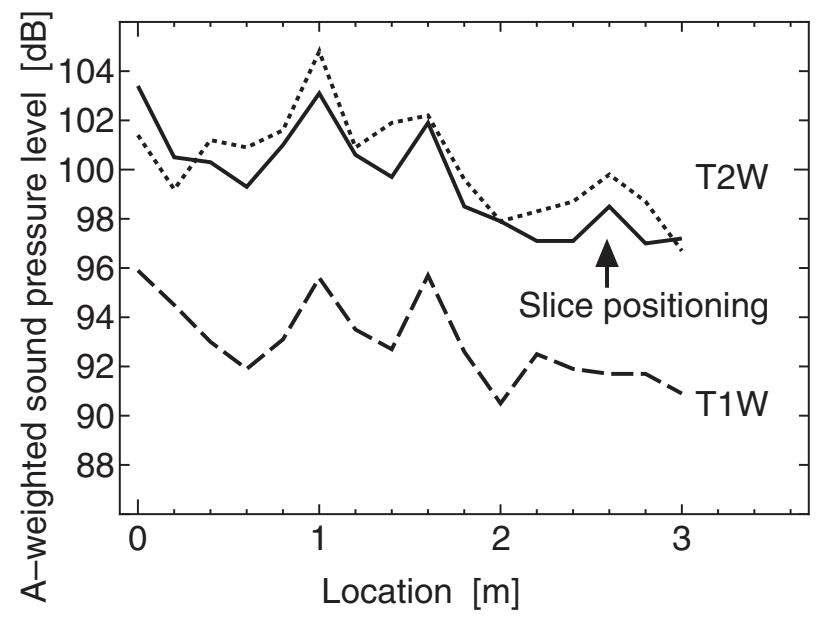

Fig. 3 Distribution of A-weighted sound pressure level on patient's table of MRI equipment.

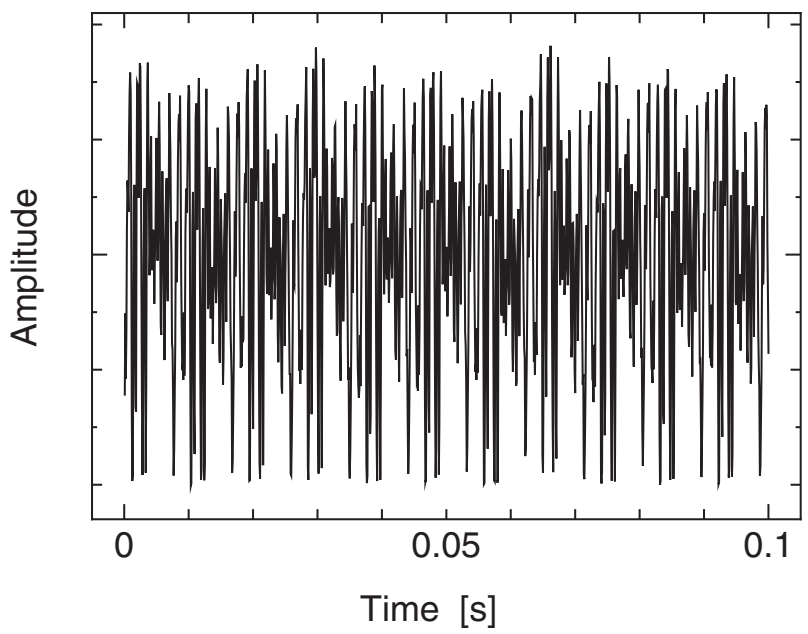

Fig. 4 Waveform in time domain at the gantry center during slice positioning.

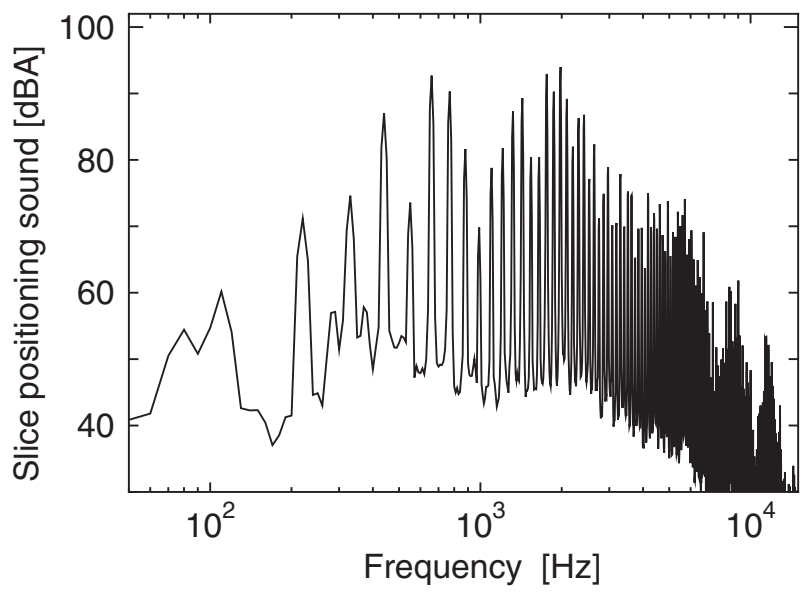

Fig. 5 A-weighted sound pressure level in frequency domain at the gantry center during slice positioning.

distribution pattern during slice positioning resembled the pattern during T2W. Because the position of the microphone array is different, the values for $\mathrm{T} 1 \mathrm{~W}$ and $\mathrm{T} 2 \mathrm{~W}$ are different from those in reference [3].

The waveform in the time domain of slice positioning is shown in Fig. 4, and the waveform in the frequency domain obtained by FFT is shown in Fig. 5. The equivalent continuous A-weighted sound pressure level during 100 seconds was $103.1 \mathrm{dBA}$, and the peak in the frequency domain was $94.0 \mathrm{dBA}$ at $1980 \mathrm{~Hz}$.

The A-weighted sound pressure level obtained by onethird octave analysis during slice positioning between $0 \mathrm{~m}$ (the gantry center) and $3 \mathrm{~m}$ is shown in Fig. 6. The A-weighted sound pressure level of one-third octave band center frequency was the highest at $1.6 \mathrm{kHz}(98.8 \mathrm{~dB})$. The patient is exposed to a high sound pressure level regardless of the part of the body under examination.

\section{Conclusions}

We first analyzed the sound of slice positioning occur- 


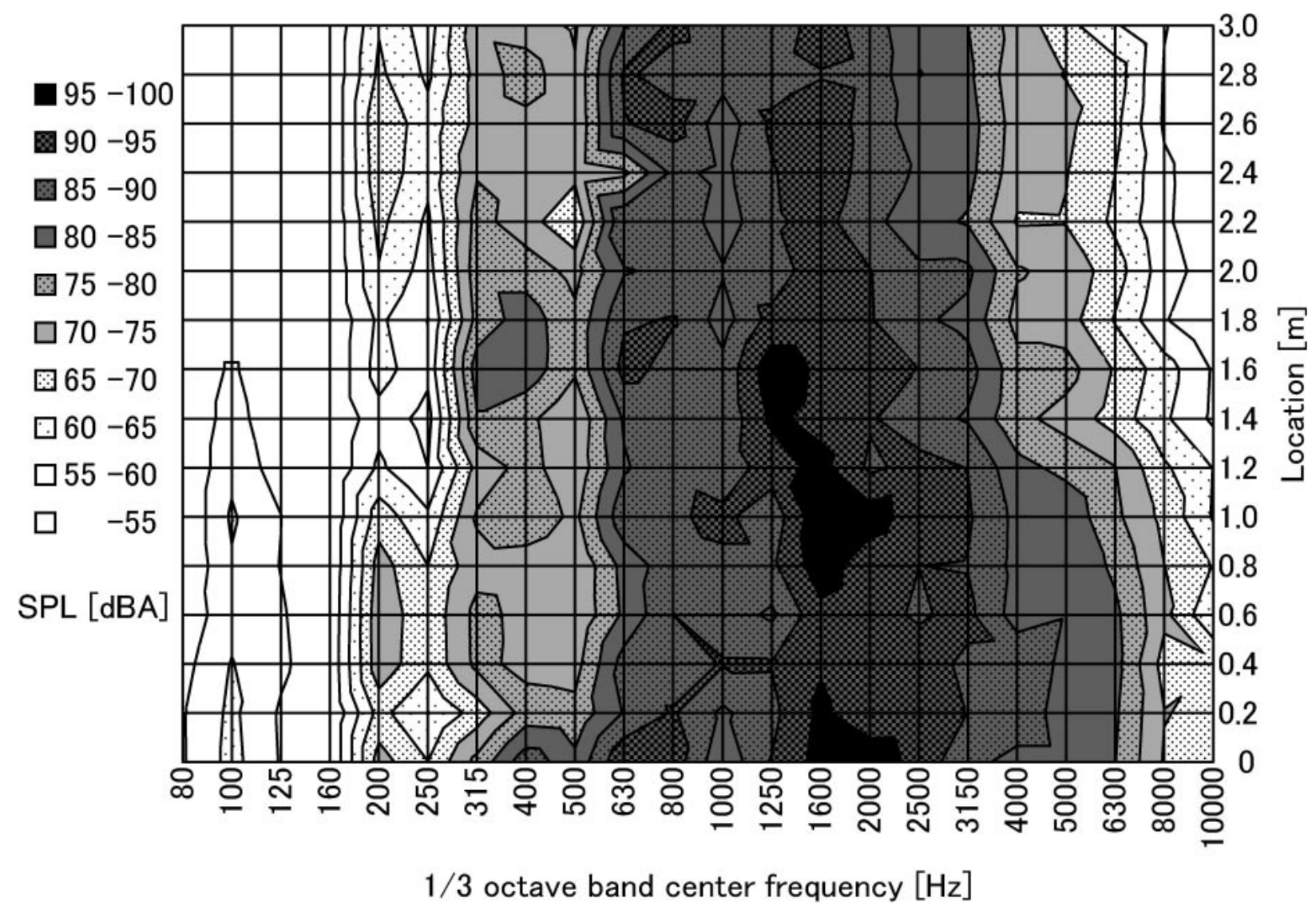

Fig. 6 Distribution of A-weighted sound pressure level obtained by one-third octave analysis during slice positioning.

ring in the first stage of the MRI examination. Second we compared it with the sound produced during T1W and T2W. It was found that the level of noise during slice positioning was almost the same as the level of T2W. Slice positioning is always performed for a short time at the beginning of each MRI examination. The patient is suddenly exposed to the sound. The patient may be startled by this sound and the psychological effect should not be underestimated. To prevent the patient from refusing further diagnosis and treatment, the patient should receive careful explanation in advance concerning the MRI examination and agree to the procedure. When other MRI models are measured in the future, it will be necessary to measure not only the noise level produced during
$\mathrm{T} 1 \mathrm{~W}, \mathrm{~T} 2 \mathrm{~W}$ and other sequences, but also the noise level produced during slice positioning.

\section{References}

[1] A. Moelker, A. J. J. Maas and P. M. T. Pattynama, "Verbal communication in MR environments: Effect of MR system acoustic noise on speech understanding," Radiology, 232, 107113 (2004).

[2] K. Yagi, S. Itoh and K. Muto, "Estimation of distribution measuring of noises in the MRI device operation," Proc. Internoise, N264 (2003).

[3] K. Muto and K. Yagi, "The measurement of the A-weighted sound pressure levels in the MRI diagnosis room," J. Acoust. Soc. Jpn. (J), 61, 5-13 (2005). 